Original article

\title{
Clinical management and outcome assessment of generalized anxiety disorder or panic disorder in refractory gastro-esophageal reflux disease: Evidence from a prospective interventional study of benzodiazepines and sertraline
}

\author{
Anuja Pradeep ${ }^{\mathrm{a}}$, Asha Susan Geoji ${ }^{\mathrm{a}}$, Athira Elezebath George ${ }^{\mathrm{a}}$, V. Athira ${ }^{\mathrm{a}}$, Dilip Chandrasekhar ${ }^{\mathrm{a}, *}$, \\ Saju Xavier ${ }^{\mathrm{b}}$, Jishnu Narayanan ${ }^{\mathrm{c}}$
}

${ }^{a}$ Department of Pharmacy Practice, Al Shifa College of Pharmacy, Perinthalmanna, Kerala, 679325, India

${ }^{\mathrm{b}}$ Department of Gastroenterology, KIMS Alshifa Hospital Pvt. Ltd, Perinthalmanna, Kerala, India

${ }^{\mathrm{c}}$ Department of Psychiatry, KIMS Alshifa Hospital Pvt Ltd, Perinthalmanna, Kerala, India

\section{A R T I C L E I N F O}

\section{Keywords:}

Benzodiazepines

Generalized anxiety disorder

Panic disorder

Refractory gastro-esophageal reflux disease

Sertraline

\begin{abstract}
A B S T R A C T
Objective: The aim of the study was to investigate the relationship between Gastro-esophageal reflux diseases (GERD) related symptoms and psychological symptomatology, as well as clinically diagnosed generalized anxiety disorder (GAD) or panic disorder (PD) and effectiveness of Sertraline and benzodiazepines in controlling these conditions.

Methodology: A 6 months prospective study was conducted in gastroenterology outpatient department of a tertiary care referral hospital. Refractory GERD was diagnosed by assessing proton pump inhibitor failure over 4 week trial of standard doses of PPIs. Therapy with Benzodiazepines and Sertraline was initiated in patients with refractory GERD having panic and anxiety symptoms associated with refractory GERD. Effectiveness of the therapy was measured using panic and agoraphobia scale and Hamilton anxiety scale. Reduction in the severity of GERD symptoms was assessed using GERD - Health related quality of life scale.

Results: The occurrence of PD or GAD in patients with refractory GERD in our sample was found to be $68 \%$ and $32 \%$ respectively. There was a significant decrease in the score of GERD HRQOL after the administration of sertraline and benzodiazepines when compared to the score of GERD HRQOL before administration of interventional drugs $(\mathrm{p}=0.001)$.

Conclusion: Our study investigated the role of anxiety and panic in refractory GERD and their effect on quality of life. The results indicated that quality of life of patients were highly improved as indicated by severity scores after administration with sertraline and benzodiazepines. The novel therapy of sertraline and benzodiazepines are highly effective in controlling reflux like symptoms and coexisting anxiety and panic disorder in refractory GERD.
\end{abstract}

\section{Introduction}

Gastro-esophageal reflux disease (GERD) is a common condition that occurs due to retrograde movement of gastric contents and causing troublesome symptoms or complications, occurring at least 2 times per week, with an adverse effect on an individual's well-being. ${ }^{1}$ The prevalence of weekly GERD symptoms reported from population-based studies worldwide is approximately $13 \%$, albeit subjected to geographic variation. $^{2}$ In India, the prevalence of GERD has been gradually increasing which attributed to the growing economics and consequently change in the lifestyle. The prevalence of GERD was $22.2 \%$ in southern India. The diagnosis and treatment strategies to be adopted for appropriate management of GERD remains a challenge despite advances in understanding its etiopathogenesis. ${ }^{3}$ Acid regurgitation and heartburn are the major symptoms in GERD and is associated with increasing health care costs and significant impact in the quality of life of these patients. $^{1}$

Acid suppressive therapy with proton pump inhibitors for a period of 4-8 weeks is deemed to be the gold standard treatment for GERD and offers rapid rates of esophageal mucosal healing and ample control of

\footnotetext{
* Corresponding author. Dept of Pharmacy Practice, Al shifa college of Pharmacy, Poonthavanam P.0, Perinthalmanna, Kerala, India, 67932.

E-mail address: dillu7@gmail.com (D. Chandrasekhar).
} 
the symptoms. ${ }^{4}$ Estimates reports a partial or complete lack of response to standard once daily dose of PPIs in about $40 \%$ of patients with heartburn. ${ }^{5}$ Failure to respond to PPI therapy has now become the most common presentation of GERD in gastrointestinal practice. ${ }^{6}$ Refractory gastro esophageal reflux disease (GERD) can be defined as the presence of typical reflux symptoms that do not respond to either once or twice daily PPI for 4 weeks. ${ }^{7}$ The prime approach in those who have failed standard PPI therapy is to assess the medication adherence and adequacy of dietary and lifestyle modifications, followed by careful escalation or administration of an alternate PPI. ${ }^{8}$

When these measures fail, further investigations are required which include evaluations for functional or structural defects in the esophagus. In addition to these structural defects, psychological factors including anxiety, panic and depression, can also develop in GERD patients, and require further assessment. ${ }^{9}$ Psychological factors and psychiatric disorders may similarly influence and exacerbate gastrointestinal disorders, such as GERD. ${ }^{10,11}$ So, it is necessary to explore refractory GERD and its relationship with psychological factors, especially because the role of psychological co morbidity and emotional stress in PPI failure has been scarcely studied. ${ }^{12,13}$ Investigators have evaluated the role of tricyclic antidepressants or SSRIs to influence esophageal perception. Antidepressants may modulate esophageal sensitivity at the central nervous system and/or sensory afferents level, potentially benefitting symptomatic patients. Similarly, a serotonin reuptake inhibitor, is effective in patients with esophageal symptoms (chest pain, dysphagia, heartburn, and/or regurgitation) associated with esophageal contraction abnormalities. ${ }^{14,15}$ Thus appropriate use of antianxiety medications such as benzodiazepines and sertraline may result in the reduction of panic and anxiety symptoms and consequent alleviation of GERD symptoms. Gastro-esophageal reflux disease (GERD), with symptoms demonstrated to impair quality of life, appears to show important variation in its prevalence. ${ }^{16}$ Evidence suggests that psychological co-morbidity is one of the critical factors that impair the QOL of individuals with GERD, but the underlying pathophysiological mechanisms remain unclear. $^{9}$

Thus evaluating the relationship between GERD and psychological comorbidities is helpful in determining an appropriate treatment regimen and thereby improving QOL of these patients. Thus our study aimed to evaluate the underlying psychological symptoms associated with refractory GERD and determine the effectiveness of sertraline and benzodiazepines to mitigate the distress and improve the QOL of the patients.

\section{Methodology}

This prospective interventional study of six months duration commencing from January 2016 to July 2017 was conducted in a tertiary care hospital of Malabar region in South India. The study protocols were approved by the ethical and research committee of the hospital and was registered under institutional ethics committee.120 outpatients in gastroenterology department with GERD symptoms were assessed for the response to standard regimen of proton pump inhibitors. The diagnosis of GERD was made out by gastroenterologist based on clinical features. The GERD health related quality of life questionnaire (GERD-HRQOL) which is a quantitative method of measuring symptom severity in GERD, was used to diagnose GERD. The scale has 11 items, which focus on heartburn symptoms, dysphagia, medication effects and the patient's present health condition, thereby surveying symptomatic outcomes and therapeutic effects in patients with GERD. Each item is scored from 0 to 5, with a higher score indicating a better QOL. As an elementary step in the research, failure of response to PPIs was assessed using GERD-HRQOL and 80 patients were found to have refractory GERD. The diagnosis of GERD was
Table 1

Socio-demographic factors of patients.

\begin{tabular}{|c|c|c|c|}
\hline & $\begin{array}{l}\% \text { of patients } n \\
\text { score }\end{array}$ & ERD-HRQOL & $P$ value \\
\hline \multicolumn{4}{|l|}{ DEMOGRAPHICS } \\
\hline Age & \multicolumn{2}{|c|}{$28 \%$ (age between 35 and 45) } & 0.2508 \\
\hline \multirow[t]{2}{*}{ Gender } & Male & $52 \%$ & 0.4110 \\
\hline & Female & $48 \%$ & \\
\hline \multirow[t]{2}{*}{ BSA } & Normal BSA & $58.9 \%$ & 0.0001 \\
\hline & Abnormal BSA & $41.1 \%$ & \\
\hline BMI & \multicolumn{2}{|l|}{$49.23 \%$} & 0.00001 \\
\hline \multicolumn{4}{|l|}{ HABIT } \\
\hline \multirow[t]{2}{*}{ Alcohol } & \multicolumn{2}{|c|}{ Alcoholic $\quad 32 \%$} & 0.00176 \\
\hline & Non-alcoholic & $68 \%$ & \\
\hline \multirow{2}{*}{ Smoking } & Smokers & $45.46 \%$ & 0.228 \\
\hline & Non-smokers & $54.54 \%$ & \\
\hline SYMPTOMS & \multicolumn{2}{|c|}{$\begin{array}{l}\text { Number of patients with higher GERD- } \\
\text { HRQOL score }\end{array}$} & \\
\hline Heartburn & \multicolumn{2}{|l|}{52} & 0.02894 \\
\hline Regurgitation & \multicolumn{2}{|l|}{47} & 0.023331 \\
\hline \multicolumn{4}{|l|}{ OTHER DISEASES } \\
\hline Diabetes Mellitus & \multicolumn{2}{|l|}{19} & 0.00003 \\
\hline Hypertension & \multicolumn{2}{|l|}{24} & 0.194208 \\
\hline \multicolumn{4}{|l|}{ OTHERS } \\
\hline Medication intake & \multicolumn{2}{|l|}{53} & 0.001 \\
\hline Food habits & \multicolumn{2}{|l|}{19} & 0.12 \\
\hline
\end{tabular}

based on clinical features and endoscopy. The gold standard is $\mathrm{pH}$ metry which has not been done since the focus is on clinical symptoms and response of these symptoms to intervention. These patients have probably panic disorder but masquerading as GERD and will be missed unless panic symptomology is probed. Patients were filtered in pursuance of inclusion criteria, comprising those diagnosed with refractory GERD by the lack of clinical response after 2 weeks of $20 \mathrm{mg}$ of omeprazole or equivalent and patients above 18 years without any chronic diseases like hypertension, diabetes mellitus, heart failure, chronic kidney disease, chronic obstructive pulmonary disease. Those patients with concurrent use of contraceptives, current or past history of bipolar, schizophrenia, psychotic, or obsessive-compulsive disorder and treatment with any drug with psychotropic activity. Patients above 70 years of age and patients with conditions like gastric stasis, obstruction, connective tissue disorder or use of drugs, alcohol or tobacco which can lead to refractory GERD are excluded from the study. History from patients and bystanders were collected and the response on follow up questioning was done for reliability. The refractory GERD was confirmed and severity assessment was executed with GERD health related quality of life questionnaire (GERD-HRQOL). The clinical outcomes such as panic disorder and generalized anxiety disorder were cliniciandiagnosed, based on ICD 10 diagnostic criteria. Primary and secondary endpoints were followed after 3 months. Among 80 patients with refractory GERD, 65 patients met the diagnostic criteria for GAD or PD, whose distribution was further estimated. The score of anxiety and panic was measured using Hamilton anxiety scale and panic and agoraphobia scale. The therapy with Benzodiazepines and sertraline was initiated in patients with refractory GERD having panic and anxiety symptoms. Consecutive escalation and tapering of dose and maintenance therapy was initiated in all patients. The dosage regimen of sertraline and benzodiazepine was designed based on theoretical evidence. Sertraline was initially administered at $25 \mathrm{mg}$ daily and increased after 2 week to $50 \mathrm{mg}$ daily. If response was not satisfactory dose was increased in steps of $25 \mathrm{mg}$ at intervals of at least 1 week to maximum of $100 \mathrm{mg}$.Initial dose of clonazepam was $0.25 \mathrm{mg}$ orally 2 times per day. The dose was increased in increments of $0.125 \mathrm{mg}-0.25 \mathrm{mg} 2$ times per day every 7 days until panic disorder is 
controlled or until side effects make further increases undesirably. Clonazepam was tapered after 1 month. The dose was decreased in $0.125 \mathrm{mg}$ decrements and stopped over 1 month. Sertraline was continued for 3-6 months. No complications were reported during the study. The primary and secondary endpoints were followed. Various comorbidities that act as risk factors for GERD including age, sex, gain in BMI, alcohol consumption and tobacco smoking were assessed and statistical conclusions were drawn. SPSS version 24.0 was used for data analysis and the level of significance was set at $5 \%$ with p value <

0.05 being considered as statistically significant for drawing inferences. Differences in categorical and continuous variables were tested with Student's t-tests. ANOVA technique for comparison for scores under different variables and Correlation analysis for testing the independence of quantitative variables. ANOVA was used to check whether BMI have any effect on GERD HRQOL score and further multiple comparison between the BMI levels was carried out using $t$-test.

\section{Results}

Sample of 120 patients were identified and followed up in the study of whom, 80 had refractory GERD. Evaluation revealed a total of 65 patients with psychiatric symptomatology, who were included in the study and provided with the interventional drugs. The psychiatric evaluation concluded that generalized anxiety disorder and panic disorder are the major psychiatric comorbidities that occur in the study population. Consequently a differential distribution of these were estimated. Patient demographics and clinical characteristics are shown in Table 1 which includes age, gender, consumption of cigarettes, hypertension and food habits. Total score of GERD-HRQOL was highest among male patients.Association between higher body surface area and refractory GERD symptoms was investigated. $86 \%$ of the refractory GERD patients had a normal body surface area (BSA) of $1.9 \mathrm{~m}^{2}$ and $14 \%$ of patients had an abnormal BSA of more than $1.9 \mathrm{~m}^{2}$. Among the refractory GERD patients with abnormal

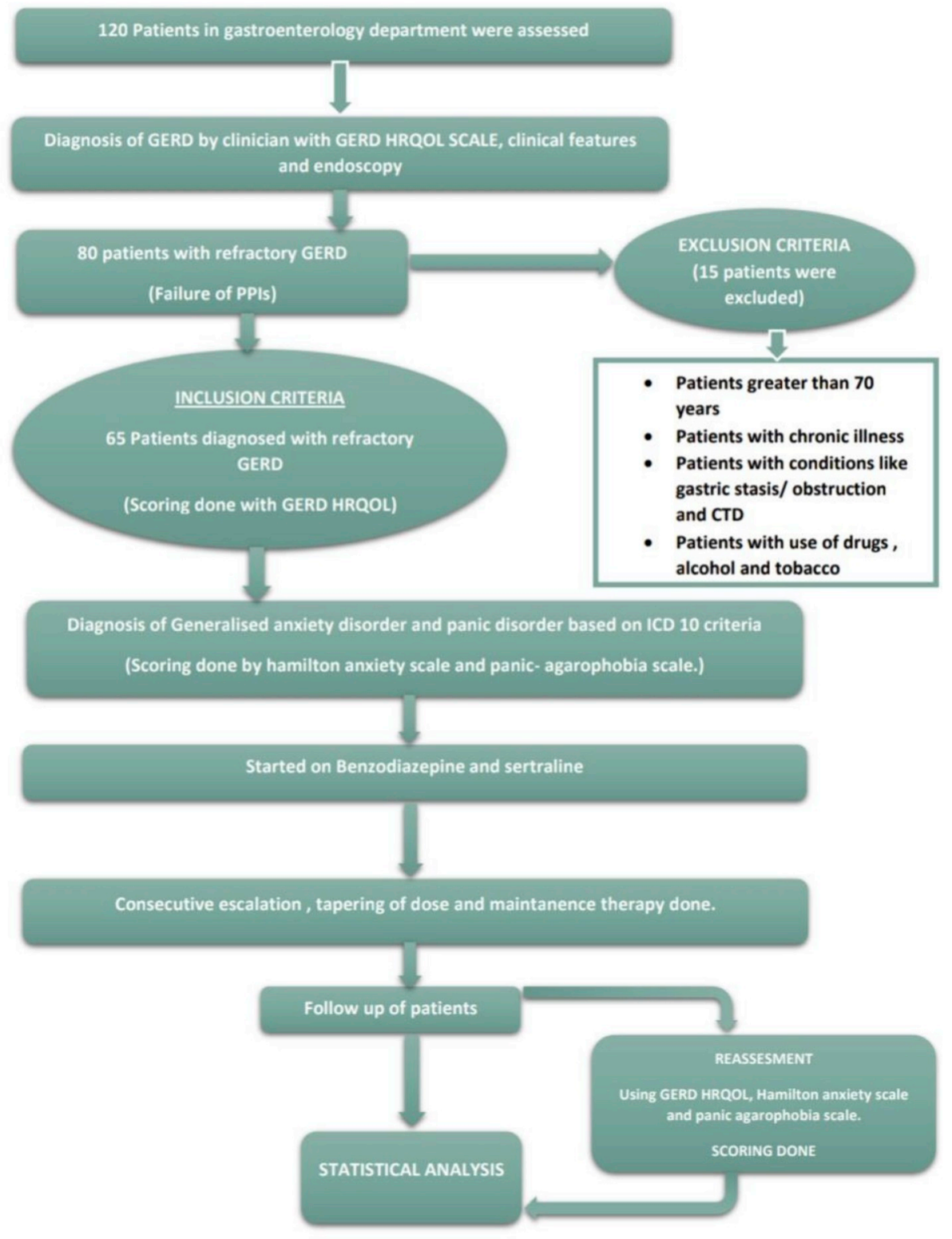

Flow chart showing methodology. 


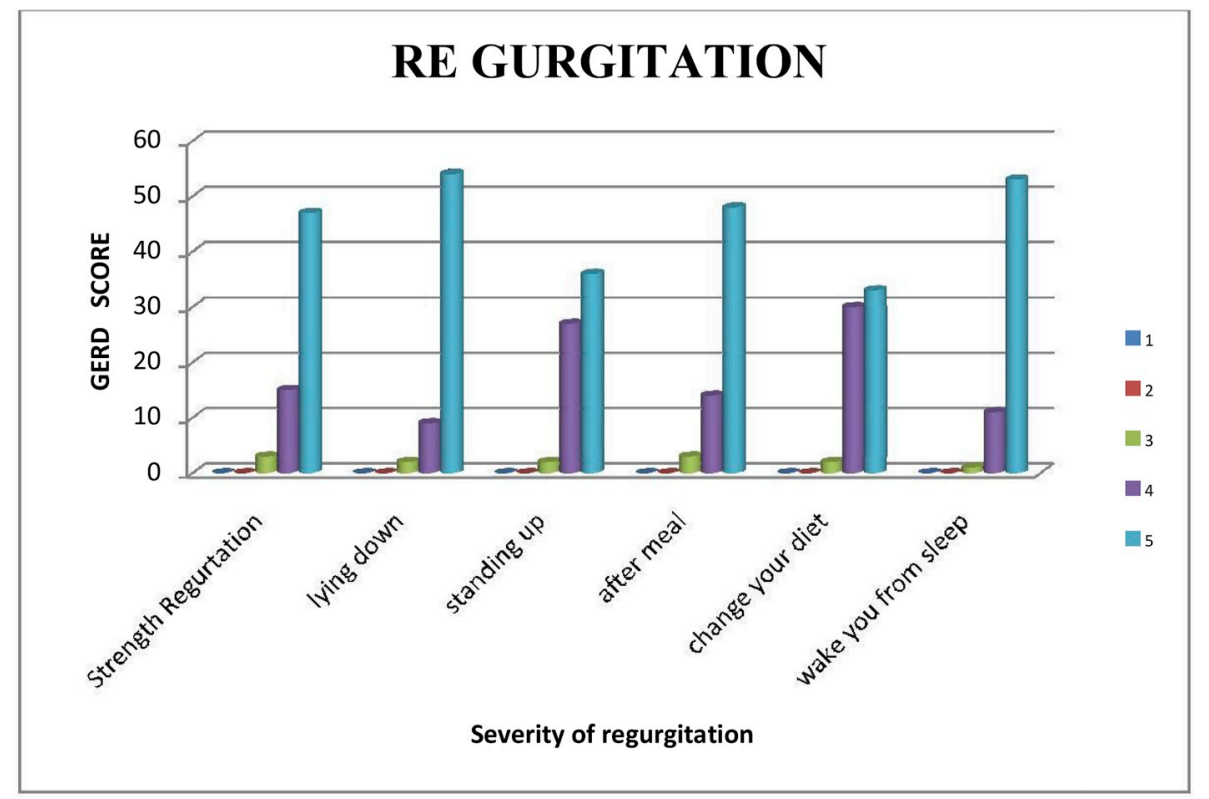

Fig. 1. Severity of regurgita tion among GERD patients.

Regurgitationshows a statistical significance in contributing to the severi ty of GERD symptoms $(\mathrm{p}=0.023331)$.

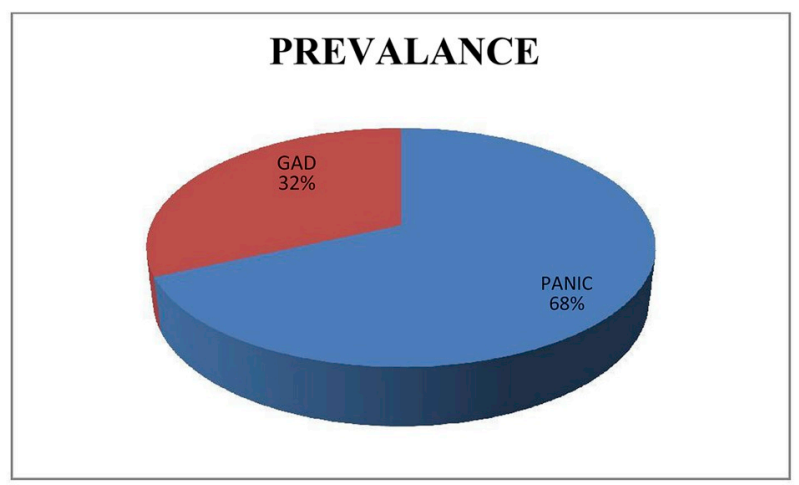

Fig. 2. Distribution of GAD and PD in refractory GERD.

BSA, about $88.88 \%$ of patients were males and $11.12 \%$ were females. Among 65 patients, $49.23 \%$ had BMI within the range of 19.5-25.5. BMI significantly influences the GERD HRQL score (f $=75.64972$; $\mathrm{p}=0.00001$ ) and the disease severity. The effect of BMI on total score significantly differs among the four categories, $<19.5$ and $19.5-25.5(\mathrm{t}$ stat $=-10.2065 ; \mathrm{p}=0.0001), 19.5-25.5$ and $25.5-28.5(\mathrm{t}$ stat $=-11.3554 ; \mathrm{p}=0.00001), 25.5-28.5$ and $>28.5(\mathrm{t}$ stat $=-2.50686 ; \mathrm{p}=0.0009524)$.Patients with refractory GERD had a higher BMI, were less likely to be physically active and more likely to report psychological symptomatology. Among the total of 33 male patients with refractory GERD, about $68 \%$ of patients were non-alcoholic and $32 \%$ were alcoholic. Alcohol significantly influences GERD score ( $\mathrm{p}=0.00176$ ). In case of smoking, $54.54 \%$ were non-smokers and $45.46 \%$ were smokers and no significant differences were observed with respect to smoking among the male patients $(\mathrm{p}=0.228)$ with refractory GERD. The development of reflux was higher in DM patients. 19 refractory GERD patients had DM. These patients scored between 65 and 70 in GERD-HRQL scale.

The most frequently reported symptoms of GERD were heartburn and was present in 52 patients. Heartburn shows a statistical significance in the severity of GERD symptoms $(\mathrm{p}=0.02894)$. Similar, regurgitation also shows a statistical significance in contributing to the severity of GERD symptoms(p $=0.023331$ ) (Fig. 1). Out of 65 patients, 53 were at higher score which shows that medication intake significantly affects quality of life of patients. Most of the patients adhered to the medication given and showed improved quality of life. The distribution of panic disorder and generalized anxiety disorder in patients with refractory GERD was found to be $68 \%$ and $32 \%$. Among the patients with panic disorder in refractory GERD, $52 \%$ were males and $48 \%$ were females. In generalized anxiety disorder, $48 \%$ of patients were males and $52 \%$ were females.

The Pearson correlation coefficient between scores of Panic and GERD was found to be +0.2 . Hence these two variables showed positive correlation. The score of Panic and agoraphobia scale showed positive correlation scores of GERD HRQOL (Fig. 2). Correlation was significant with $\mathrm{p}$ value $<0.001$. The Pearson correlation coefficient between scores of Hamilton anxiety scale and GERD was +0.15 . Hence these two variables were positively correlated, as Anxiety scores increased, the score of GERD HRQOL also increased (Fig. 3). Correlation was significant with $\mathrm{p}$ value $<0.001$. According to Pearson Correlation, there is a significant decrease in the score of panic agoraphobia scale and Hamilton anxiety scale after the administration of standard doses of Sertraline and Benzodiazepines (interventional drugs) when compared to the scores before administration of interventional drugs. There is also significant decrease in the score of GERD HRQOL after the administration of standard doses of Sertraline and Benzodiazepines (interventional drugs) when compared to the score of GERD HRQOL before administration of interventional drugs (Fig. 4 and Fig. 5). 


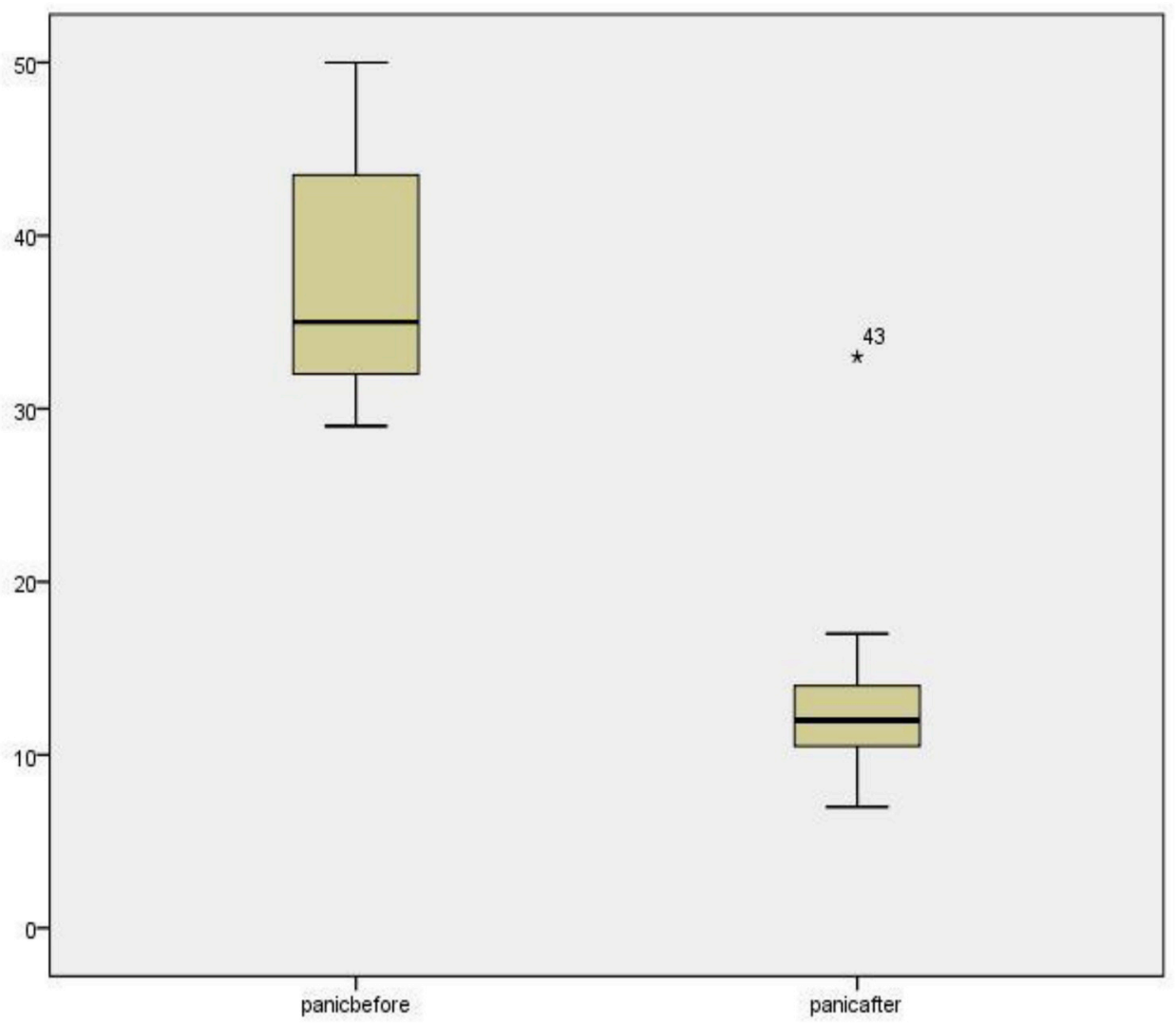

\section{$(\mathrm{t}$ stat $=19.856 ; \mathrm{p}=0.001)$.}

Fig. 3. Relationship between pre-interventional and post interventional scores of GERD.

\section{Discussion}

The aims of the present study was to investigate the relationship between GERD-related symptoms and anxiety disorder and to assess the effectiveness of sertraline and clonazepam in controlling these conditions. The study indicated that panic disorder and anxiety disorder is common in GERD and probably makes them refractory to PPI therapy. From the assessment of the study population, we found out that heart burn and regurgitation are the major clinical presentations in refractory GERD associated with psychiatric symptomatology. Ram Dickmann et al., ${ }^{17}$ in his study concluded that heart burn was significantly less prevalent in those who fully responded to PPI once daily when compared to patients who failed PPI once daily and twice daily thus indicating heart burn to be significantly associated with increased occurrence in refractory GERD patients. Various reflux symptoms like heartburn and regurgitation are thus found to have significant association in refractory GERD with psychiatric comorbidities.

Another study evaluated the frequency of reflux symptoms in patients with a diagnosed psychiatric disorder and assessed the potential risk factors for symptom occurrence. ${ }^{18}$ The presence of the psychiatric condition was found to be the prime factor associated with increased reflux in the above-mentioned study. The concurrent esophageal dysfunction was proposed to be the etiological factor for increased reflux in psychiatric disorders.

Our study indicated a significant difference between baseline and endpoint scores of both panic and anxiety following the administration of interventional drugs. A study conducted by Hartono JL et al. ${ }^{19}$ found that anxiety can lead to non-erosive reflux disease (NERD), which is the presence of classic GERD symptoms in the absence of esophageal mucosal injury during upper endoscopy. Psychological factors play an important role in patients with GERD as per this study and have been shown to decrease QOL in these patients considerably. A cross sectional study following a similar pattern to our study conducted by Jansson C et al. has indicated that anxiety can worsen the symptoms of reflux. ${ }^{20}$ The study revealed a high prevalence of depressive, anxiety and sleep disorders among GERD patients. In our study, the panic and agoraphobia scale (PAS) was used to measure the severity of illness in patients with panic disorder (with or without agoraphobia). Moreover, Hamilton Anxiety Rating Scale (HAM-A) was used to measure the severity of anxiety symptoms by measuring both psychic anxiety (mental agitation and psychological distress) and somatic anxiety (physical complaints related to anxiety). But in the afore mentioned study, rating scales such as HADS were used for screening of these disorders rather than diagnoses by psychiatrists.

You Z-H et al. ${ }^{21}$ suggested that GERD increases the risk of developing depressive, anxiety, and sleep disorders negatively influencing their QOL. The study following a co relational and longitudinal study design indicated frequent occurrence of psychiatric disorders among GERD patients. However our study was limited to identifying the differential distribution of GAD and PD among patients with refractory 




Fig. 4. Relationship between pre-interventional and post interventional scores of GERD.

GERD. Previous studies have analyzed the roles of both anxiety and depression symptoms on the incidence of GERD, as well as their negative impact on QOL in patients with GERD. The results suggested that the incidence of GERD was correlated with anxiety and depression, and QOL of patients with GERD was reduced statistically. ${ }^{18}$ Both sertraline and benzodiazepines was found to reduce the scores of panic or anxiety respectively in the patients and thereby reduces the total severity score of GERD. Various studies have demonstrated that sertraline is an effective and well tolerated treatment of anxiety disorders with the anxiolytic benefit extending to both psychic and somatic anxiety symptoms. ${ }^{22}$ Benzodiazepine is also effective in the treatment of anxiety disorders, because of their rapid onset of action and their efficacy with a favorable side effect profile. ${ }^{23}$ However, there is a high risk of benzodiazepine dependence, with longer duration of benzodiazepine treatment. A thorough risk assessment guides optimal management and the necessity for referral. However, the chances of dependence was minimized in our study by gradual benzodiazepine withdrawal and maintenance treatment with SSRI. Tricyclic antidepressants and selective serotonin reuptake inhibitors have shown to improve esophageal pain by reducing psychological and mental factors, which are known to be capable of inducing GERD symptoms. ${ }^{24}$

Mental health specialists in the field of psycho-gastroenterology can aid in the management of esophageal hypersensitivity, which can drive the symptom experience of a PPI non responder. Considerations for comorbid anxiety and depression in this population require careful assessment and treatment. ${ }^{25}$ Furthermore, symptomatic improvement in the psychiatric symptoms is associated with parallel improvement in quality of life and occupational functioning of GERD patients.

\section{Conclusion}

The study has shown that anxiety and panic disorder is very frequent in patients with refractory GERD symptoms. Our study's findings, in the context of an interventional study, provide primary evidence about the usefulness of targeting psychological symptoms for treatment of refractory GERD. Also, these findings is an indication of the key role of psychological factors on failure of PPIs and symptom persistence. This study showed that Sertraline and Benzodiazepines are effective in reducing the overall severity of GERD by controlling the psychological symptomatology. The overall improvement in the quality of life of the patients achieved by these medicines are thus attributed to the improvement in psychological wellbeing. The study regarding the correlation of panic and anxiety with GERD is a novel concept and can be drawn out onto a wider canvas so as to estimate a stronger evidence. Further prospective interventional studies are required to confirm the impacts of treating of psychological symptoms associated with refractory GERD.

\section{Declaration of competing interest}

The authors declare no conflict of interest. 


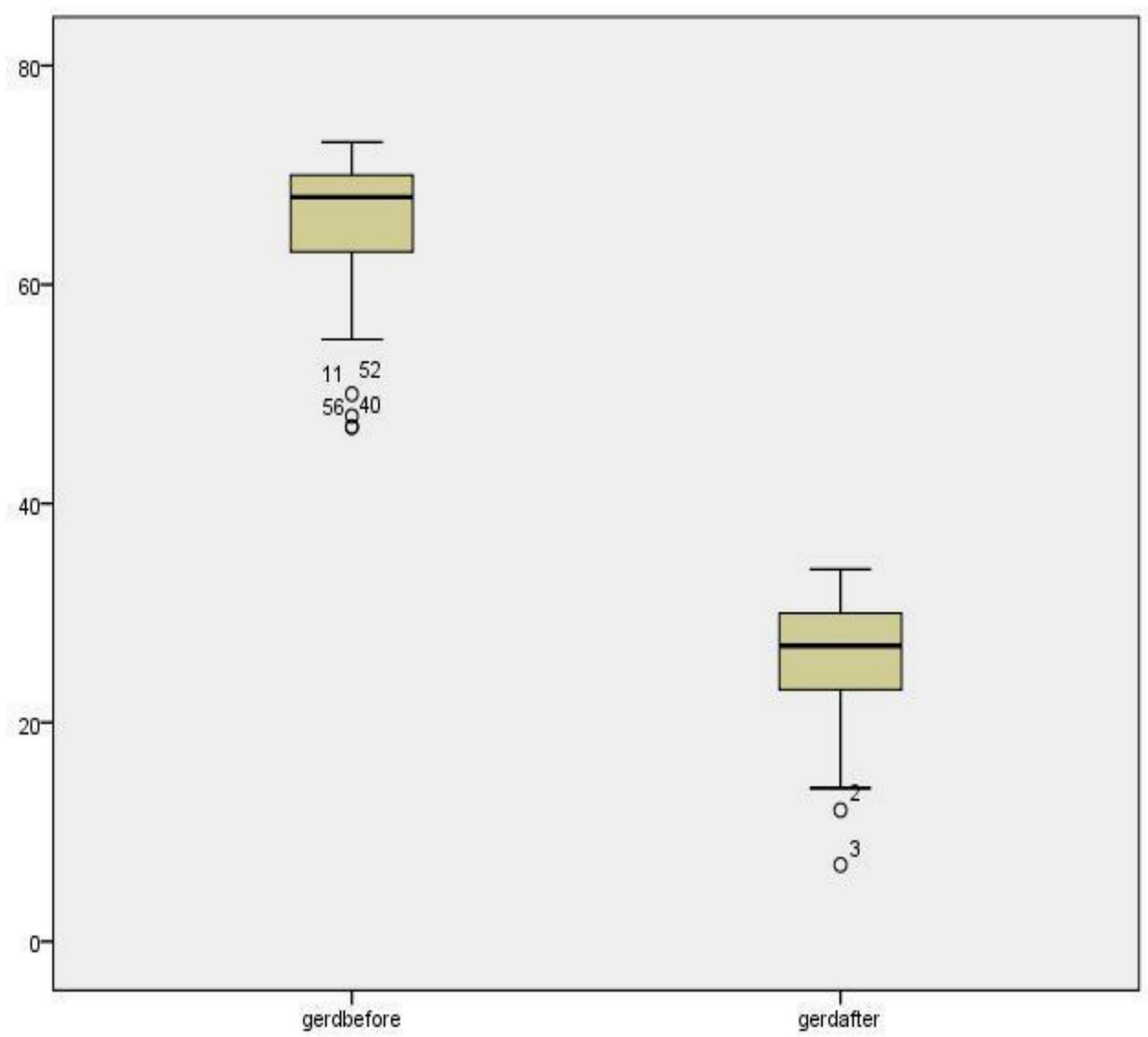

\section{(t stat $=39.47 ; \mathrm{p}=0.001)$}

Fig. 5. Relationship between pre-interventional and post interventional scores of GERD.

\section{References}

1. Lo Wai-Kit, Mashimo Hiroshi. Establishing the diagnosis of GERD. Failed Anti-reflux Therapy. Springer Cham; 2017:13-18.

2. Eusebi LH, Ratnakumaran R, Yuan Y, Solaymani-Dodaran M, Bazzoli F, Ford AC. Global prevalence of, and risk factors for, gastro-oesophageal reflux symptoms: a meta-analysis. Gut. 2017 Feb 23;2016 gutjnl-

3. Pritchett J, Aslam M, Slaughter J, Ness R, Garrett C, Vaezi M. Efficacy of esophageal impedance/pH monitoring in patients with refractory gastroesophageal reflux disease, on and off therapy. Clin Gastroenterol Hepatol. 2009;7(7):743-748.

4. Fass R. Proton-pump inhibitor therapy in patients with gastro-oesophageal reflux disease: putative mechanisms of failure. Drugs. 2007;67:1521-1530.

5. Hershcovici T, Fass R. Management of gastroesophageal reflux disease that does not respond well to proton pump inhibitors. Curr Opin Gastroenterol. 2010:26:367-378.

6. Nunez-Rodriguez MH, Miranda Sivelo A. Psychological factors in gastroesophageal reflux disease measured by scl-90-R questionnaire. Dig Dis Sci. 2008;53:3071-3075

7. Kahrilas PJ, Shaheen NJ, Vaezi MF. American gastroenterological association medical position statement on the management of gastroesophageal reflux disease. Gastroenterology. 2008;135:1383-1391.

8. Subramanian Charumathi Raghu, Triadafilopoulos George. Refractory gastroesophageal reflux disease. Gastroenterol Rep. 2015;3(1):41-53.

9. Yang XJ, Jiang HM, Hou XH, Song J. Anxiety and depression in patients with gastroesophageal reflux disease and their effect on quality of life. World J Gastroenterol. 2015;21(14):4302-4309.

10. Kamolz T, Velanovich V. Psychological and emotionalaspectsofgastro-esophageal reflux disease. Dis Esophagus. 2002;15:199-203.

11. Bhatia V, Tandon RK. Stressandthe gastro-intestinal tract. J Gastroenterol Hepatol. 2005;20:332-339.

12. Avidan B, Sonnenberg A, Giblovich H, Sontag SJ. Refluxsymptomsare associated withpsychiatric disease. Aliment Pharmacol Ther. 2001;15:1907-1912.

13. Haug TT, Mykletun A, DahlA A. The prevalence ofnauseainthecommunity: psychological,socialandsomatic factors. Gen Hosp Psychiatr. 2002;24:81-86.

14. Prakash C, Clouse RE. Long-term outcome from tricyclic antidepressant treatment of functional chest pain. Dig Dis Sci. 1999 Dec;44(12):2373-2379.

15. Clouse RE, Lustman PJ, Eckert TC, Ferney DM, Griffith LS. Low-dose trazodone for symptomatic patients with esophageal contraction abnormalities. A double-blind, placebo-controlled trial. Gastroenterology. 1987;92(4):1027-1036.

16. Hampel H, Abraham NS, El-Serag HB. Meta-analysis: obesity and the risk for gastroesophageal reflux disease and its complications. Ann Intern Med. 2005 Feb;143(3):199.

17. Dickman R, Boaz M, Aizic S, Beniashvili Z, Fass R, Niv Y. comparison of clinical characteristics of patients with gastroesophageal reflux disease who failed proton pump inhibitor therapy versus those who fully responded. $J$ Neuro Gastroenterol Motil. 2011;17(4):387-394.

18. Avidan B, Sonnenberg A, Giblovich H, Sontag SJ. Reflux symptoms are associated with psychiatric disease. Aliment Pharmacol Therapeut. 2001;15(12):1907-1912.

19. Hartono JL, Mahadeva S, Goh KL. Anxiety and depression in various functiona gastrointestinal disorders: do differences exist? J Dig Dis. 2012;13:252-257.

20. Jansson C, Nordenstedt H, Wallander MA, Johansson S, et al. Severe gastro-oesophageal reflux symptoms in relation to anxiety, depression and coping in a population-based study. Aliment Pharmacol Ther. 2007;26:683-691.

21. You Z-H, et al. Risk of psychiatric disorders following gastroesophageal reflux disease: a nationwide population-based cohort study. Eur J Intern Med. 2015;26(7):534-539.

22. Allgulander Christer, Dahl Alv A, Austin Carol, et al. Efficacy of sertraline in a 12week trialfor generalized anxiety disorder. Am J Psychiatr. 2004;161:1642-1649.

23. Cloos Jean-Marc, Ferreira Valérie. Current use of benzodiazepines in anxiety disorders. Curr Opin Psychiatr. 2009;22(1):90-95.

24. Faruqui AA. Anxiety induced refractory gastrointestinal disorders. J Liver Res Disord Ther. 2017;3(4):00062

25. Riehl ME, Chen JW. The proton pump inhibitor nonresponder: a behavioral approach to improvement and wellness. Curr Gastroenterol Rep. 2018 Jul 1;20(7):34. 